\title{
Interaction of Acoustic and Thermal Modes in the Vibrationally Relaxing Gases. Acoustic Cooling
}

\author{
A. PeRElomova* \\ Gdańsk University of Technology, Faculty of Applied Physics and Mathematics \\ G. Narutowicza 11/12, 80-952 Gdańsk, Poland
}

(Received December 1, 2011; in final form February 1, 2013)

\begin{abstract}
The dynamic equation which governs an excess temperature associated with the thermal mode in vibrationally relaxing gas is derived. The nonlinear transfer of acoustic energy to the energy of the thermal mode in a relaxing gas causes slow variation of temperature with time. The final dynamic equation is instantaneous. All types of sound, including aperiodic, may be considered as an acoustic source of corresponding heating or cooling. The study considers sound with frequencies much larger than the inverse time of the thermodynamic relaxation. In the nonequilibrium regime, if standard attenuation is neglected, gas temperature decreases with time. Examples concern heating and cooling caused by periodic in time sound and an impulse. The influence of standard viscosity, thermal conductivity, and heat withdrawal is briefly discussed.
\end{abstract}

DOI: 10.12693/APhysPolA.123.681

PACS: 43.35.Fj, 43.25.Ed

\section{Introduction.}

Basic equations and starting points

The establishment of nonequilibrium molecular physics began in the sixties due to the laser revolution in physics and chemistry. The interest to the hydrodynamics of the nonequilibrium fluids is governed primarily by the fact that this is one of the new fields of modern hydrodynamics. It is now passing through the stage of formulating the fundamental equations and for revealing new physical effects. Nonequilibrium gases include, among other, the interstellar medium, upper atmosphere, discharge plasma. Interest in nonequilibrium phenomena in the physics of gases was firstly connected with studies of anomalous dispersion and absorption of ultrasonics waves. The reason for these anomalies is usually the mechanism of retarded energy exchange between the internal and translational degrees of freedom of the molecules [1-3]. A number of problems relating to the nonlinear effects and rate processes in gases with internal relaxation, have been studied previously. In this context, the contributions due to Chu [4], Parker [5], Clarke and McChesney [6] are worth mentioning.

This paper is devoted to one important type of nonlinear phenomena, caused by sound. The nonlinear loss in acoustic energy in the standard thermoviscous fluid, which enlarges the background temperature, is called acoustic heating $[7,8]$. A rate of temperature increase is proportional to the sound intensity and overall attenuation in a fluid. It was firstly pointed out by Molevich [9] that the nonlinear exchange of energy between sound and the thermal mode may lead to cooling instead

*e-mail: anpe@mif.pg.gda.pl of heating in the nonequilibrium gas, if the standard attenuation is small. The possibility of an anomaly in the other nonlinear phenomenon caused by dominative sound in a vibrationally excited molecular gas, streaming, was also pointed out there. The flow of this secondary vortex motion may occur in the opposite direction compared to that in the equilibrium gas.

The method worked out by the author allows to derive instantaneous dynamic equations for sound and non-wave modes accounting for their interaction. We start from the linear determination of modes as specific types of gas motion whose steady but nonequilibrium state is maintained by pumping energy into the vibrational degrees of freedom by power $I$ and a heat withdrawal from the translational degrees of freedom of power $Q$ (both $I$ and $Q$ refer to unit mass) (Sect. 2). This is necessary for correct decomposition of equations governing sound and the thermal mode accounting for the interactions of modes (Sect. 3). The relaxation equation for the vibrational energy per unit mass should complete the system of conservation equations in the differential form. It takes the form

$$
\frac{\mathrm{d} \varepsilon}{\mathrm{d} t}=-\frac{\varepsilon-\varepsilon_{\mathrm{eq}}(T)}{\tau}+I .
$$

The equilibrium value of the vibrational energy at the given temperature $T$ is denoted by $\varepsilon_{\mathrm{eq}}(T)$, and $\tau(\rho, T)$ marks the vibrational relaxation time. The quantity $\varepsilon_{\mathrm{eq}}(T)$ in the case of a system of harmonic oscillators equals

$$
\varepsilon_{\text {eq }}(T)=\frac{\hbar \Omega}{m\left[\exp \left(\hbar \Omega / k_{\mathrm{B}} T\right)-1\right]},
$$

where $m$ is the mass of a molecule, $\hbar \Omega$ is the magnitude of the vibrational quantum, $k_{\mathrm{B}}$ is the Boltzmann constant. Equation (2) is valid over the temperatures, where one can neglect anharmonic effects, i.e., below the characteristic temperatures, which are fairly high for most molecules [1-3]. The mass, momentum and energy equa- 
tions for a thermoviscous flow in a vibrationally relaxing gas read

$$
\begin{aligned}
& \frac{\partial \rho}{\partial t}+\nabla \cdot(\rho \boldsymbol{v})=0, \\
& \rho\left[\frac{\partial \boldsymbol{v}}{\partial t}+(\boldsymbol{v} \cdot \boldsymbol{\nabla}) \boldsymbol{v}\right] \\
& \quad=-\nabla p+\eta \Delta \boldsymbol{v}+\left(\zeta+\frac{\eta}{3}\right) \boldsymbol{\nabla}(\boldsymbol{\nabla} \cdot \boldsymbol{v}), \\
& \rho\left[\frac{\partial(e+\varepsilon)}{\partial t}+(\boldsymbol{v} \cdot \boldsymbol{\nabla})(e+\varepsilon)\right]+p(\boldsymbol{\nabla} \cdot \boldsymbol{v}) \\
& \quad=\chi \Delta T+\rho(I-Q)+\zeta(\boldsymbol{\nabla} \cdot \boldsymbol{v})^{2} \\
& \quad+\frac{\eta}{2}\left(\frac{\partial v_{i}}{\partial x_{k}}+\frac{\partial v_{k}}{\partial x_{i}}-\frac{2}{3} \delta_{i k} \frac{\partial v_{l}}{\partial x_{l}}\right)^{2},
\end{aligned}
$$

where $\boldsymbol{v}$ denotes velocity of a fluid, $\rho, p$ are density and pressure, $e$ marks internal energy per unit mass of translation motion of molecules, $\zeta, \eta$ are bulk and shear viscosities (all supposed to be constants), $x_{i}$ denote space coordinates. Besides Eq. (2), two thermodynamic functions $e(p, \rho), T(p, \rho)$ complete the system (3). Thermodynamics of ideal gases provides equalities

$$
e(p, \rho)=\frac{p}{(\gamma-1) \rho}=\frac{R}{\mu(\gamma-1)} T(p, \rho),
$$

where $\gamma$ is the isentropic exponent without account for vibrational degrees of freedom, $R$ is the universal gas constant, and $\mu$ is the molar mass of a gas.

\section{Planar motions of infinitely small amplitude and their decomposition}

Let us consider a motion of infinitely small amplitude of a gas in the case $\eta=0, \zeta=0, \chi=0, Q=$ const, $I=$ const. The flow is supposed to be planar along axis $O x$. We will discuss the influence of thermal conductivity, viscosity and the kind of function $Q(T)$ in Sect. 3.4 below. In this subsection, the results will be verified by including of the first partial derivative of $Q$ with respect to temperature $T$. Considering every quantity $q$ as a sum of unperturbed value $q_{0}$ (in absence of the background flows, $v_{0}=0$ ) and its variation $q^{\prime}$, one readily rearranges the governing equations of momentum, energy balance and continuity into the form

$$
\begin{aligned}
& \frac{\partial v^{\prime}}{\partial t}+\frac{1}{\rho_{0}} \frac{\partial p^{\prime}}{\partial x}=0 \\
& \frac{\partial p^{\prime}}{\partial t}+\gamma p_{0} \frac{\partial v^{\prime}}{\partial x}-(\gamma-1) \rho_{0} \frac{\varepsilon^{\prime}}{\tau} \\
& \quad+(\gamma-1) \rho_{0} T_{0} \Phi_{1}\left(\frac{p^{\prime}}{p_{0}}-\frac{\rho^{\prime}}{\rho_{0}}\right)=0 \\
& \frac{\partial \rho^{\prime}}{\partial t}+\rho_{0} \frac{\partial v^{\prime}}{\partial x}=0, \\
& \frac{\partial \varepsilon^{\prime}}{\partial t}+\frac{\varepsilon^{\prime}}{\tau}-T_{0} \Phi_{1}\left(\frac{p^{\prime}}{p_{0}}-\frac{\rho^{\prime}}{\rho_{0}}\right)=0
\end{aligned}
$$

where

$$
\Phi_{1}=\left(\frac{C_{v}}{\tau}+\frac{\varepsilon-\varepsilon_{\mathrm{eq}}}{\tau^{2}} \frac{\mathrm{d} \tau}{\mathrm{d} T}\right)_{0}
$$

is the quantity evaluated at $p_{0}, T_{0}$, and $C_{v}=\mathrm{d} \varepsilon_{\text {eq }} / \mathrm{d} T$.
The expansion in series of equations of state (4) was used to express perturbations of translation temperature and internal translational energy per unit mass

$$
e^{\prime}=\frac{p_{0}}{(\gamma-1) \rho_{0}}\left(\frac{p^{\prime}}{p_{0}}-\frac{\rho^{\prime}}{\rho}\right)=\frac{R}{\mu(\gamma-1)} T^{\prime} .
$$

The last equation in the set (5) follows from Eq. (1):

$$
\begin{aligned}
\frac{\partial \varepsilon^{\prime}}{\partial t} & +\frac{\varepsilon^{\prime}}{\tau}=\left(\frac{C_{v}}{\tau}+\frac{\varepsilon-\varepsilon_{\mathrm{eq}}}{\tau^{2}} \frac{\mathrm{d} \tau}{\mathrm{d} T}\right)_{0} T^{\prime} \\
& =T_{0} \Phi_{1}\left(\frac{p^{\prime}}{p_{0}}-\frac{\rho^{\prime}}{\rho_{0}}\right) .
\end{aligned}
$$

The relaxation time in the most important cases may be thought as a function of temperature accordingly to Landau and Teller, $\tau(T)=A \exp \left(B T^{-1 / 3}\right)$, where $A$ and $B$ are some positive constants [1-3]. The Landau-Teller dependence of relaxation time provides negative values of $\mathrm{d} \tau / \mathrm{d} T$.

Studies of fluid motions of infinitely-small amplitudes start usually with representing of all perturbations as a sum of planar waves, where $\tilde{q}(k) \exp (\mathrm{i} \omega(k) t)$ is the Fourier-transforms of any perturbation $q^{\prime}$ :

$$
q^{\prime}(x, t)=\int_{-\infty}^{\infty} \tilde{q}(k) \exp (\mathrm{i}(\omega t-k x)) \mathrm{d} k+\text { c.c. }
$$

The approximate roots of dispersion equation for both acoustic branches, progressive in the positive and negative directions of axis $O x$, are well-known under the simplifying condition $\omega \tau \gg 1$, which restricts consideration by the high-frequency sound $[3,10]$ :

$$
\begin{aligned}
& \omega_{1}=c k+\frac{\mathrm{i}}{2} \frac{(\gamma-1)^{2} T_{0}}{c^{2}} \Phi_{1}, \\
& \omega_{2}=-c k+\frac{\mathrm{i}}{2} \frac{(\gamma-1)^{2} T_{0}}{c^{2}} \Phi_{1},
\end{aligned}
$$

where $c=\sqrt{\frac{\gamma R T_{0}}{\mu}}=\sqrt{\frac{\gamma p_{0}}{\rho_{0}}}$ denotes the infinitely small-signal sound speed in an ideal uniform gas. The last term in the both dispersion relations manifests amplification of sound in the nonequilibrium regime (if $\Phi_{1}<0$ ) which does not depend on wave number $k$. The amplification effect increases with enlargement of $|\mathrm{d} \tau / \mathrm{d} T|$ and vibrational nonequilibrium $m\left(\varepsilon-\varepsilon_{\text {eq }}\right) / k_{\mathrm{B}} T$. We will consider $\left|\Phi_{1} T_{0} / c^{3} k\right| \ll 1$. This condition provides a weak distortion of the sound wave (caused by attenuation or amplification) over its period. The two last roots of dispersive equation, estimated without limitation $\omega \tau \gg 1$, sound

$$
\begin{aligned}
& \omega_{3}=\mathrm{i}\left(\frac{1}{\tau}+\frac{(\gamma-1)\left(\gamma+c^{2} k^{2} \tau^{2}\right) T_{0}}{c^{2}\left(1+c^{2} k^{2} \tau^{2}\right)} \Phi_{1}\right), \\
& \omega_{4}=0 .
\end{aligned}
$$

Third, non-wave mode, determined by $\omega_{3}$, comes from the vibrational relaxation. The fourth root exists in any planar flow of a fluid, not necessarily relaxing or attenuating, it represents the thermal, or entropy, mode. In equilibrium gas, this type of non-wave motion specifies isobaric increase in the background temperature and correspondent variation in its density. It is well-established that the nonlinear losses in acoustic energy in a gas with 
typical thermoviscous attenuation lead to the heating of the background, and by means of that, influence on the sound velocity in a fluid. The last two roots manifest slow varying and stationary, non-wave motions of a gas. They are of importance, among other applications, in studies of the variations in the background after sound passing.

The overall velocity, pressure, density and internal energy are also a sum of specific parts: $v^{\prime}(x, t)=$ $\sum_{n=1}^{4} v_{n}^{\prime}(x, t)$, and so on. In accordance to the roots $(10),(11)$, the Fourier-transforms of dynamic variables may be represented as a linear combination of four specific Fourier-transforms of excess density $\tilde{\rho}_{1}, \tilde{\rho}_{2}, \tilde{\rho}_{3}, \tilde{\rho}_{4}$ as follows:

$$
\begin{aligned}
\tilde{\rho} & =\sum_{n=1}^{4} \tilde{\rho}_{n}, \tilde{v}=\sum_{n=1}^{4} \tilde{v}_{n}=\sum_{n=1}^{4} \omega_{n} \tilde{\rho}_{n} / k / \rho_{0}, \tilde{p}=\sum_{n=1}^{4} \tilde{p}_{n} \\
& =\sum_{n=1}^{4} \omega_{n}^{2} \tilde{\rho}_{n} / k^{2}, \tilde{\varepsilon}=\sum_{n=1}^{4} \tilde{\varepsilon}_{n} \\
& =\frac{T_{0} \Phi_{1}}{\rho_{0} c^{2}} \sum_{n=1}^{2} \tilde{\rho}_{n}\left(\frac{\gamma \omega_{n}^{2}}{k^{2}}-c^{2}\right) /\left(\mathrm{i} \omega_{n}\right) \\
& +\frac{T_{0} \Phi_{1}}{\rho_{0} c^{2}} \tilde{\rho}_{3}\left(\frac{\gamma \omega_{3}^{2}}{k^{2}}-c^{2}\right) /\left(\mathrm{i} \omega_{3}+1 / \tau\right) \\
& +\frac{\tau T_{0} \Phi_{1}}{\rho_{0} c^{2}} \tilde{\rho}_{4}\left(\frac{\gamma \omega_{4}^{2}}{k^{2}}-c^{2}\right) .
\end{aligned}
$$

The links in the $(x, t)$ space follow from Eqs. (12) and roots of dispersion relations, Eqs. (10), (11). It is easy to establish the operators $\tilde{d}_{1}^{1}, \tilde{d}_{2}^{1}, \tilde{d}_{3}^{1}, \tilde{d}_{4}^{1}$ applying in the Fourier-transform space in order to decompose from the vector of overall perturbations only one constituent of overall excess density, for example, corresponding to the rightwards progressive sound

$$
\tilde{d}_{1}^{1} \tilde{v}+\tilde{d}_{2}^{1} \tilde{p}+\tilde{d}_{3}^{1} \tilde{\rho}+\tilde{d}_{4}^{1} \tilde{\varepsilon}=\tilde{\rho}_{1} .
$$

Equation (13) in fact contains four algebraic equations determining four unknown quantities uniquely. The matrix of 4 rows, projecting the vector of perturbations into the vector of specific excess densities, has the form as follows:

$$
\begin{aligned}
& \left(\begin{array}{cccc}
\tilde{d}_{1}^{1} & \tilde{d}_{2}^{1} & \tilde{d}_{3}^{1} & \tilde{d}_{4}^{1} \\
\tilde{d}_{1}^{2} & \tilde{d}_{2}^{2} & \tilde{d}_{3}^{2} & \tilde{d}_{4}^{2} \\
\tilde{d}_{1}^{3} & \tilde{d}_{2}^{3} & \tilde{d}_{3}^{3} & \tilde{d}_{4}^{3} \\
\tilde{d}_{1}^{4} & \tilde{d}_{2}^{4} & \tilde{d}_{3}^{4} & \tilde{d}_{4}^{4}
\end{array}\right) \cdot\left(\begin{array}{c}
\tilde{v} \\
\tilde{p} \\
\tilde{\rho} \\
\tilde{\varepsilon}
\end{array}\right) \equiv \tilde{D} \cdot\left(\begin{array}{c}
\tilde{v} \\
\tilde{p} \\
\tilde{\rho} \\
\tilde{\varepsilon}
\end{array}\right)=\left(\begin{array}{c}
\tilde{\rho}_{1} \\
\tilde{\rho}_{2} \\
\tilde{\rho}_{3} \\
\tilde{\rho}_{4}
\end{array}\right) \cdot \tilde{D} ; \\
& \left(\begin{array}{c}
\tilde{d}_{1}^{1} \\
\tilde{d}_{2}^{1} \\
\tilde{d}_{3}^{1} \\
\tilde{d}_{4}^{1}
\end{array}\right)=\left(\begin{array}{c}
\frac{\rho_{0}}{2 c}-\frac{\mathrm{i}(\gamma-1)^{2} \rho_{0} T_{0}}{2 c^{4} k} \Phi_{1} \\
\frac{1}{2 c^{2}}-\frac{\mathrm{i}(\gamma-1)(\gamma-3) T_{0}}{4 c^{5} k} \Phi_{1} \\
-\frac{\mathrm{i}(\gamma-1) T_{0}}{2 c^{3} k} \Phi_{1} \\
-\frac{\mathrm{i}(\gamma-1) \rho_{0}}{2 c^{3} k \tau}
\end{array}\right) ; \\
& \left(\begin{array}{c}
\tilde{d}_{1}^{2} \\
\tilde{d}_{2}^{2} \\
\tilde{d}_{3}^{2} \\
\tilde{d}_{4}^{2}
\end{array}\right)=\left(\begin{array}{c}
-\frac{\rho_{0}}{2 c}-\frac{\mathrm{i}(\gamma-1)^{2} \rho_{0} T_{0}}{2 c^{4} k} \Phi_{1} \\
\frac{1}{2 c^{2}}+\frac{\mathrm{i}(\gamma-1)(\gamma-3) T_{0}}{45^{5} k} \Phi_{1} \\
\frac{\mathrm{i}(\gamma-1) T_{0}}{2 c^{3} k} \Phi_{1} \\
\frac{\mathrm{i}(\gamma-1) \rho_{0}}{2 c^{3} k \tau}
\end{array}\right) ;
\end{aligned}
$$

$$
\begin{aligned}
& \left(\begin{array}{c}
\tilde{d}_{1}^{3} \\
\tilde{d}_{2}^{3} \\
\tilde{d}_{3}^{3} \\
\tilde{d}_{4}^{3}
\end{array}\right)=\left(\begin{array}{c}
\frac{\mathrm{i}(\gamma-1)^{2} \rho_{0} T_{0}}{c^{4} k} \Phi_{1} \\
-\frac{(\gamma-1) T_{0} \tau}{c^{4}} \Phi_{1} \\
\frac{(\gamma-1) T_{0} \tau}{c^{2}} \Phi_{1} \\
\frac{(\gamma-1) \rho_{0}}{c^{2}}-\frac{(\gamma-1)^{2} T_{0} \rho_{0} \tau}{c^{4}} \Phi_{1}
\end{array}\right) ; \\
& \left(\begin{array}{c}
\tilde{d}_{1}^{4} \\
\tilde{d}_{2}^{4} \\
\tilde{d}_{3}^{4} \\
\tilde{d}_{4}^{4}
\end{array}\right)=\left(\begin{array}{c}
0 \\
-\frac{1}{c^{2}}+\frac{(\gamma-1) T_{0} \tau}{c^{4}} \Phi_{1} \\
1-\frac{(\gamma-1) T_{0} \tau}{c^{2}} \Phi_{1} \\
-\frac{(\gamma-1) \rho_{0}}{c^{2}}+\frac{(\gamma-1)^{2} T_{0} \rho_{0} \tau}{c^{4}} \Phi_{1}
\end{array}\right) .
\end{aligned}
$$

The terms of $\tilde{D}$ are evaluated within accuracy up to terms involving $\Phi_{1}^{1}$ and $(1 /(c k \tau))^{1}$ inclusively. The analogous matrix operator $D$, operating in the $(x, t)$ space, may be easily derived, taking in mind that $(-\mathrm{i} k)^{-1}$ corresponds to the operator $\int \mathrm{d} x$. Limits of integration depend on the physical context of the problem. Application of any row of matrix $D$ on the overall vector of perturbations decomposes the specific excess density, correspondent to this row. Analogously, application of these rows individually on the system (5) itself results in dynamic equation of specific excess density, correspondent to this row. For example, application of the first and last row of the $D$ on the system (5) yields dynamic equations for the excess densities of rightwards progressive sound (in the limit $c k \tau \gg 1)$ and the entropy mode, relatively

$$
\frac{\partial \rho_{1}^{\prime}}{\partial t}+c \frac{\partial \rho_{1}^{\prime}}{\partial x}+\frac{(\gamma-1)^{2} T_{0}}{2 c^{2}} \Phi_{1} \rho_{1}^{\prime}=0, \quad \frac{\partial \rho_{4}}{\partial t}=0 .
$$

That obviously coincides to the roots of dispersion equation $\omega_{1}$ and $\omega_{4}$ established by Eq. (10), (11). In the other words, the system of linear Eqs. (5) is projected uniquely into four independent equations for every specific mode. Projecting is in fact a certain way of linear combination of equations in order to keep one specific quantity in the linear part of equations (in this study, the specific excess density. The choice of reference specific variables may look different) and reduce all specific quantities correspondent to other modes there. It bases on linear links of the field perturbations which in fact determine every mode as well as dispersion relations.

\section{Governing equations in a weakly nonlinear flow}

\subsection{Decomposing of specific equations by means of projecting}

Account for the nonlinear terms of the second order in Eqs. (1), (4) yields the leading order series

$$
\begin{aligned}
T^{\prime} & =T_{0}\left(\frac{p^{\prime}}{p_{0}}-\frac{\rho^{\prime}}{\rho_{0}}+\frac{\rho^{\prime 2}}{\rho_{0}^{2}}-\frac{p^{\prime} \rho^{\prime}}{p_{0} \rho_{0}}\right) \\
\frac{\mathrm{d} \varepsilon^{\prime}}{\mathrm{d} t} & =-\frac{\varepsilon^{\prime}}{\tau}+T_{0}\left(\frac{1}{\tau^{2}} \frac{\mathrm{d} \tau}{\mathrm{d} T}\right)_{0} \varepsilon^{\prime}\left(\frac{p^{\prime}}{p_{0}}-\frac{\rho^{\prime}}{\rho_{0}}\right) \\
& +T_{0} \Phi_{1}\left(\frac{p^{\prime}}{p_{0}}-\frac{\rho^{\prime}}{\rho_{0}}+\frac{\rho^{\prime 2}}{\rho_{0}^{2}}-\frac{p^{\prime} \rho^{\prime}}{p_{0} \rho_{0}}\right)
\end{aligned}
$$




$$
\begin{aligned}
& +T_{0} \Phi_{2}\left(\frac{p^{\prime}}{p_{0}}-\frac{\rho^{\prime}}{\rho_{0}}\right)^{2}, \\
\Phi_{2} & =T_{0}\left[-\frac{1}{\tau^{2}} C_{v} \frac{\mathrm{d} \tau}{\mathrm{d} T}-\frac{\varepsilon_{0}-\varepsilon_{\mathrm{eq}}}{\tau^{3}}\left(\frac{\mathrm{d} \tau}{\mathrm{d} T}\right)^{2}\right. \\
& \left.+\frac{1}{2 \tau} \frac{\mathrm{d} C_{v}}{\mathrm{~d} T}+\frac{\left(\varepsilon_{0}-\varepsilon_{\mathrm{eq}}\right)}{2 \tau^{2}} \frac{\mathrm{d}^{2} \tau}{\mathrm{d} T^{2}}\right]_{0} .
\end{aligned}
$$

The governing dynamic system with account for quadratic nonlinear terms differs from (5) by the quadratic right-hand side

$$
\begin{aligned}
& \frac{\partial v^{\prime}}{\partial t}+\frac{1}{\rho_{0}} \frac{\partial p^{\prime}}{\partial x}=-v^{\prime} \frac{\partial v^{\prime}}{\partial x}+\frac{\rho^{\prime}}{\rho_{0}^{2}} \frac{\partial p^{\prime}}{\partial x}, \\
& \frac{\partial p^{\prime}}{\partial t}+\gamma p_{0} \frac{\partial v^{\prime}}{\partial x}-(\gamma-1) \rho_{0} \frac{\varepsilon^{\prime}}{\tau} \\
& +(\gamma-1) \rho_{0} T_{0} \Phi_{1}\left(\frac{p^{\prime}}{p_{0}}-\frac{\rho^{\prime}}{\rho_{0}}\right) \\
& =-v^{\prime} \frac{\partial p^{\prime}}{\partial x}-\gamma p^{\prime} \frac{\partial v^{\prime}}{\partial x}+(\gamma-1) \rho^{\prime} \\
& \times\left[\frac{\varepsilon^{\prime}}{\tau}-T_{0} \Phi_{1}\left(\frac{p^{\prime}}{p_{0}}-\frac{\rho^{\prime}}{\rho_{0}}\right)\right]-(\gamma-1) \rho_{0} \\
& \times\left[T_{0}\left(\frac{1}{\tau^{2}} \frac{\mathrm{d} \tau}{\mathrm{d} T}\right)_{0} \varepsilon^{\prime}\left(\frac{p^{\prime}}{p_{0}}-\frac{\rho^{\prime}}{\rho_{0}}\right)\right. \\
& \left.+T_{0} \Phi_{1}\left(\frac{\rho^{\prime 2}}{\rho_{0}^{2}}-\frac{p^{\prime} \rho^{\prime}}{p_{0} \rho_{0}}\right)+T_{0} \Phi_{2}\left(\frac{p^{\prime}}{p_{0}}-\frac{\rho^{\prime}}{\rho_{0}}\right)^{2}\right], \\
& \frac{\partial \rho^{\prime}}{\partial t}+\rho_{0} \frac{\partial v^{\prime}}{\partial x}=-v^{\prime} \frac{\partial \rho^{\prime}}{\partial x}-\rho^{\prime} \frac{\partial v^{\prime}}{\partial x}, \\
& \frac{\partial \varepsilon^{\prime}}{\partial t}+\frac{\varepsilon^{\prime}}{\tau}-T_{0} \Phi_{1}\left(\frac{p^{\prime}}{p_{0}}-\frac{\rho^{\prime}}{\rho_{0}}\right) \\
& =T_{0}\left(\frac{1}{\tau^{2}} \frac{\mathrm{d} \tau}{\mathrm{d} T}\right)_{0} \varepsilon^{\prime}\left(\frac{p^{\prime}}{p_{0}}-\frac{\rho^{\prime}}{\rho_{0}}\right) \\
& +T_{0} \Phi_{1}\left(\frac{\rho^{\prime 2}}{\rho_{0}^{2}}-\frac{p^{\prime} \rho^{\prime}}{p_{0} \rho_{0}}\right) \\
& +T_{0} \Phi_{2}\left(\frac{p^{\prime}}{p_{0}}-\frac{\rho^{\prime}}{\rho_{0}}\right)^{2}-v^{\prime} \frac{\partial \epsilon^{\prime}}{\partial x} .
\end{aligned}
$$

The linear projecting is fruitful in investigations of nonlinear interactions of different types of motion [11]. Contributions of all other modes in the linear part of the final dynamic equation are simply cancelled, but the nonlinear terms become distributed between equations in the proper way.

The problems of generation of the non-acoustic types of motion by the dominative sound are of major importance. From the physical point of view, the mode is dominative, when amplitudes of its perturbations are much larger than those of other modes. It is well-established that besides loss in acoustic energy, which leads to acoustic heating, representing the thermal mode, loss in acous- tic momentum takes place which results in a streaming in the standard thermoviscous nonlinear flows. Streaming exists only in the multi-dimensional flow, it is rotational motion. The thermal mode may exist in the planar flow as well. In the setting of these problems, sound is dominative as compared with other types of motion. Since the order of magnitude of secondary flows is no higher, than the squared Mach number $O\left(M^{2}\right)$ (they are typical nonlinear phenomena), the accurate account for the quadratic corrections in the dominative sound, which are of the same order, are necessary $[11,12]$.

\subsection{Nonlinear equation governing dominative sound}

Applying the row operator $\left(\begin{array}{llll}d_{1}^{1} & d_{2}^{1} & d_{3}^{1} & d_{4}^{1}\end{array}\right)$ on the both sides of equations which form the system (17), letting all nonlinear terms be acoustic correspondent to the first (progressive in the positive direction of axis $O x$ ) mode, and expressing all perturbations in the terms of specific excess densities, one can readily derive the dynamic equation governing the dominative sound within accuracy up to terms of order $M^{2}$. Its nonlinear terms may be considered as certain corrections caused by interaction of this wave with itself. The row $\left(\begin{array}{llll}d_{1}^{1} & d_{2}^{1} & d_{3}^{1} & d_{4}^{1}\end{array}\right)$ in view of $c k \tau \gg 1,\left|\Phi_{1}\right| T_{0} /\left(c^{3} k\right) \ll 1$, takes the form:

$$
\left(\begin{array}{l}
d_{1}^{1} \\
d_{2}^{1} \\
d_{3}^{1} \\
d_{4}^{1}
\end{array}\right)=\left(\begin{array}{c}
\frac{\rho_{0}}{2 c}-\frac{(\gamma-1)^{2} \rho_{0} T_{0}}{2 c^{4}} \Phi_{1} \int \mathrm{d} x \\
\frac{1}{2 c^{2}}-\frac{(\gamma-1)(\gamma-3) T_{0}}{4 c^{5}} \Phi_{1} \int \mathrm{d} x \\
-\frac{(\gamma-1) T_{0}}{2 c^{3}} \Phi_{1} \int \mathrm{d} x \\
-\frac{(\gamma-1) \rho_{0}}{2 c^{3} \tau} \int \mathrm{d} x
\end{array}\right) .
$$

The vector $\psi_{1}$ below represents the linear links for the rightwards progressive sound in the leading order, as it follows from (10), (12):

$$
\psi_{1}=\left(\begin{array}{c}
v_{1}^{\prime}(x, t) \\
p_{1}^{\prime}(x, t) \\
\rho_{1}^{\prime}(x, t) \\
\varepsilon_{1}^{\prime}(x, t)
\end{array}\right)=\left(\begin{array}{c}
\frac{c}{\rho_{0}}+\frac{(\gamma-1)^{2} \Phi_{1} T_{0}}{2 c^{2} \rho_{0}} \int \mathrm{d} x \\
c^{2}+\frac{(\gamma-1)^{2} \Phi_{1} T_{0}}{c} \int \mathrm{d} x \\
1 \\
-\frac{(\gamma-1) \Phi_{1} T_{0}}{c \rho_{0}} \int \mathrm{d} x
\end{array}\right) \rho_{1}^{\prime} .
$$

Accounting for (18), (19), and applying the row $\left(\begin{array}{llll}d_{1}^{1} & d_{2}^{1} & d_{3}^{1} & d_{4}^{1}\end{array}\right)$ on the both sides of equations from the system (17), one gets the leading order dynamic equation for the acoustic excess density

$$
\frac{\partial \rho_{1}^{\prime}}{\partial t}+c \frac{\partial \rho_{1}^{\prime}}{\partial x}-c B \rho_{1}^{\prime}=-\frac{\gamma+1}{2} \frac{c}{\rho_{0}} \rho_{1}^{\prime} \frac{\partial \rho_{1}^{\prime}}{\partial x},
$$

where

$$
B=-\frac{(\gamma-1)^{2} T_{0}}{2 c^{3}} \Phi_{1} .
$$

This equation may be easily rewritten in terms of acoustic pressure by use of specific relation between it and an excess acoustic density (Eq. (19)). The term in the right-hand side of Eq. (20) manifests the well-celebrated nonlinearity originating from nonlinearity in equation of state and hydrodynamic nonlinearity $[7,8]$, it is of order $M^{2}$. To derive coupling nonlinear equations, one requires involving the corrections in the linear definition of sound by account for terms specific for the Riemann wave [7]. They support adiabaticity of sound in the loss- 
less flow. The corrected links with accuracy up to terms of order $M^{2}$ for the rightwards progressive sound are

$$
\Psi_{1}=\left(\begin{array}{c}
\frac{c}{\rho_{0}}-\frac{c B}{\rho_{0}} \int \mathrm{d} x+\frac{(\gamma-3) c}{4 \rho_{0}^{2}} \rho_{1}^{\prime} \\
c^{2}-2 c^{2} B \int \mathrm{d} x+\frac{(\gamma-1) c^{2}}{2 \rho_{0}} \rho_{1}^{\prime} \\
1 \\
\frac{2 B c^{2}}{(\gamma-1) \rho_{0}} \int \mathrm{d} x
\end{array}\right) \rho_{1}^{\prime} .
$$

\subsection{Governing equation for the thermal mode. Acoustic heating or cooling?}

In the context of the problem, sound is dominative, and the corresponding thermal mode, caused by it, is secondary with magnitude of excess density much less than that of sound: $\left|\rho_{4, A}\right| \ll\left|\rho_{1, A}\right|$. Let us consider only the rightwards propagating sound, assuming that the leftwards one and the third mode, though may enlarge their magnitudes in time, keep small comparatively to the dominative first mode. The quadratic nonlinear corrections in the sound mode (22) should be considered in order to derive valid dynamic equation of the thermal mode. At this point, we make routine manipulations to decompose the dynamic equation for the specific excess density of the entropy mode by means of applying on the system (17) by the row $\left(d_{1}^{4} d_{2}^{4} d_{3}^{4} d_{4}^{4}\right)$ and collecting together terms of the leading order. The final equation governing acoustic heating is

$$
\frac{\partial \rho_{4}^{\prime}}{\partial t}=-\frac{2 B c}{\rho_{0}}\left(\rho_{1}^{\prime 2}+\gamma \frac{\partial \rho_{1}^{\prime}}{\partial x} \int \rho_{1}^{\prime} \mathrm{d} x\right) .
$$

The acoustic excess density in the right-hand side must itself satisfy the dynamic Eq. (20).

Equation (23) bases on the consequent subdivision of equations for different types of motions by use of their properties. It is instantaneous and applies to both periodic and aperiodic sound. The only simplifications used to simplify calculations, are $\omega_{1} \tau \gg 1,|B| \ll k, M \ll 1$. A simple estimation of acoustic source in the case of periodic sound may be done on the base of the linear equation, into which the governing equation of sound (20) rearranges in the case of the infinitely-small amplitude sound, the first one from Eqs. (15). Its simple periodic solution has the form

$$
\rho_{1}^{\prime}=M \rho_{0} \sin (c k(t-x / c)) \exp (B x) .
$$

Taking in mind that during an isobaric process of an ideal gas $T \rho=$ const, $T^{\prime}=-\left(T_{0} / \rho_{0}\right) \rho^{\prime}$, Eq. (23) with excess acoustic density in the form (24) results in the leading order to the following equality:

$$
\begin{gathered}
\left\langle\frac{\partial T_{4}^{\prime}}{\partial t}\right\rangle=-B T_{0} c(\gamma-1) M^{2} \exp (2 B x) \\
=-\frac{2 B T_{0}(\gamma-1)}{\rho_{0} c^{2}} E(x),
\end{gathered}
$$

where $\langle\phi(x, t)\rangle=\frac{c k}{2 \pi} \int_{t}^{t+2 \pi /(c k)} \phi(x, t) \mathrm{d} t$ is temporal average over sound period, $E(x)=\left\langle p_{1}^{\prime} v_{1}^{\prime}\right\rangle$, is the intensity of sound, $T_{4}^{\prime}$ is the excess background temperature, a quantity relating to the thermal mode. Note that there are acoustic periodic variations in temperature in this exam- ple. On the average, they do not influence on the slow variations of the background temperature. The right-hand side of Eq. (25) is the acoustic source of heating or cooling, its negative value for positive $B$ guarantees cooling of a gas in the non-equilibrium regime. The temperature production is positive in the equilibrium regime like it takes place in the standard thermoviscous flows. In the Newtonian fluids, the periodic acoustic excess density and correspondent heating take the form

$$
\begin{aligned}
& \rho_{1}^{\prime}=M \rho_{0} \sin (c k(t-x / c)) \exp (-\alpha x), \\
& \left\langle\frac{\partial T_{4}^{\prime}}{\partial t}\right\rangle=\frac{\alpha T_{0} c(\gamma-1) M^{2}}{2} \exp (-2 \alpha x) \\
& =\frac{\alpha T_{0}(\gamma-1)}{\rho_{0} c^{2}} E(x),
\end{aligned}
$$

where $\alpha=\frac{b k^{2}}{2 c \rho_{0}}, b=4 \eta / 3+\zeta+\chi(\gamma-1)^{2} /(\gamma R \mu)$ is the standard attenuation including the thermal one. The similarity of Eqs. (25) and (26) is obvious, if $B<0$. The nonlinear Eq. (20) has the exact solution for the sinusoidal at a transducer signal of frequency $\omega$, which is valid before forming of a saw-like wave [3]:

$$
\begin{aligned}
\rho_{1}^{\prime} & =M \rho_{0} \exp (B x) \\
& \times \sum_{n=1}^{\infty} \frac{\left.2 J_{n} n K(\exp (B x)-1)\right] \sin (n \omega(t-x / c)}{n K[\exp (B x)-1]},
\end{aligned}
$$

where $K=\frac{(\gamma+1) M \omega}{2 B c}$. The distance from the transducer, where the waveform breaks up, is

$$
x_{b}=\ln (1+1 / K) B^{-1} .
$$

The variation in temperature associated with the thermal mode, in accordance to Eq. (23), is

$$
\begin{gathered}
\left\langle\frac{\partial T_{4}^{\prime}}{\partial t}\right\rangle=-B T_{0} c(\gamma-1) M^{2} \exp (2 B x) \\
\times \sum_{n=1}^{\infty}\left[\frac{2 J_{n}(n K(\exp (B x)-1))}{n K(\exp (B x)-1)}\right]^{2} .
\end{gathered}
$$

Figure 1 shows dimensionless variations in temperature associated with the entropy mode, in a unit time for some positive and negative values of $B$.

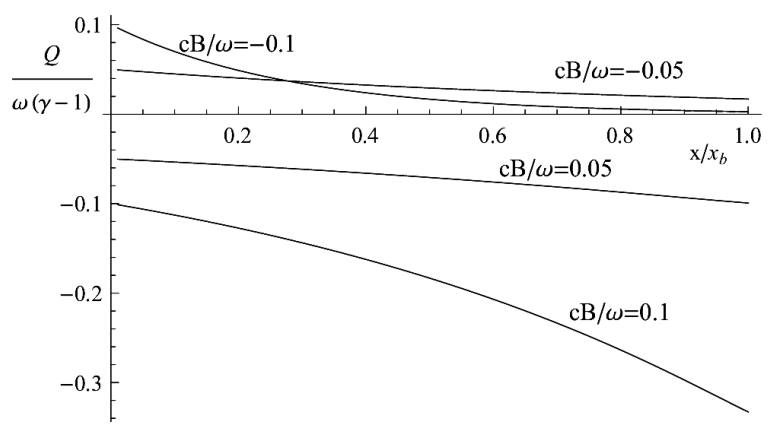

Fig. 1. Variation in temperature in unit time, $Q=$ $\frac{1}{T_{0}} \frac{\partial T_{4}^{\prime}}{\partial t}$ as a function of dimensionless distance from a transducer for $M=0.1$ in accordance to Eq. (29), before forming of the saw-like wave. 
In the case of simple impulse sound in the role of acoustic source, $\rho_{1}^{\prime}=M \rho_{0} \phi(\eta=k c(t-x / c))$, Eq. (23) is readily integrated to yield in the leading order

$$
\begin{aligned}
& T_{4}^{\prime}(\eta)=\frac{2 B T_{0} M^{2}}{k} \\
& \quad \times\left(\gamma \phi \int_{-\infty}^{\eta} \phi(\eta) \mathrm{d} \eta-(\gamma-1) \int_{-\infty}^{\eta} \phi^{2}(\eta) \mathrm{d} \eta\right) .
\end{aligned}
$$

Figure 2 illustrates the excess temperature caused by simple symmetric and asymmetric pulses. The trace after a pulse passing is the excess temperature of background, positive for negative $B$. In evaluations, $\gamma$ equals 1.4 .

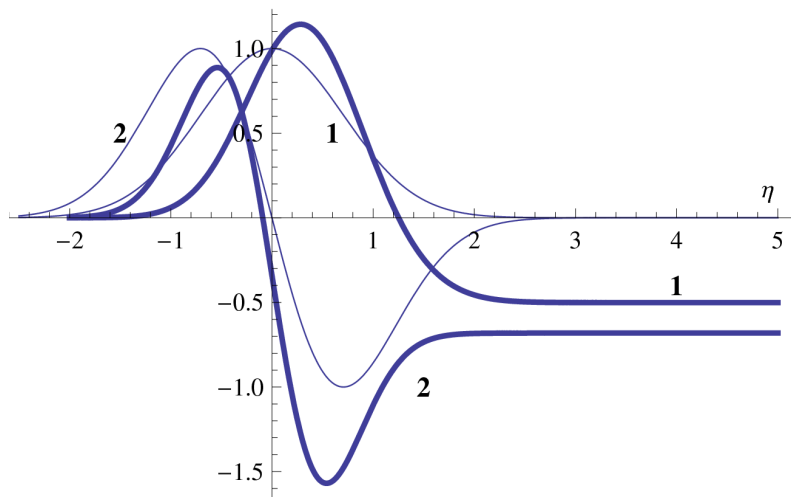

Fig. 2. Sound pulses $\phi=\exp \left(-\eta^{2}\right)(1$, thin $)$ and $\phi=$ $-\sqrt{2 e} \eta \exp \left(-\eta^{2}\right)(2$, thin $)$ and caused by them excess temperature (1, thick, and 2 , thick, relatively) $\frac{k}{2 B M^{2}} \frac{T_{4}^{\prime}}{T_{0}}$ as functions of $\eta$.

\subsection{Inclusion of viscosity, thermal conductivity and heat withdrawal $Q(T)$}

Taking into account for thermal conductivity and viscosity results in corrections in the roots of dispersion relation. Two acoustic ones, under condition $\omega \tau \gg 1$, are

$$
\begin{aligned}
& \omega_{1}=c k+\frac{\mathrm{i} b k^{2}}{2 \rho_{0}}+\frac{\mathrm{i}}{2} \frac{(\gamma-1)^{2} T_{0}}{c^{2}}\left(\Phi_{1}+Q_{T}\right), \\
& \omega_{2}=-c k+\frac{\mathrm{i} b k^{2}}{2 \rho_{0}}+\frac{\mathrm{i}}{2} \frac{(\gamma-1)^{2} T_{0}}{c^{2}}\left(\Phi_{1}+Q_{T}\right),
\end{aligned}
$$

where $Q_{T}=(\mathrm{d} Q / \mathrm{d} T)_{0}$. The third root keeps unchanged, and the last one sounds

$$
\omega_{4}=\mathrm{i} \frac{\chi k^{2}(\gamma-1)}{\gamma R \mu \rho_{0}}+\mathrm{i} \frac{(\gamma-1) Q_{T} T_{0}}{c^{2}} .
$$

The linear modes and projectors take the new form. Links for the rightwards progressive sound are as follows:

$$
\psi_{1}=\left(\begin{array}{c}
v_{1}^{\prime}(x, t) \\
p_{1}^{\prime}(x, t) \\
\rho_{1}^{\prime}(x, t) \\
\varepsilon_{1}^{\prime}(x, t)
\end{array}\right)
$$

$$
=\left(\begin{array}{c}
\frac{c}{\rho_{0}}+\frac{(\gamma-1)^{2}\left(\Phi_{1}+Q_{T}\right) T_{0}}{2 c^{2} \rho_{0}} \int \mathrm{d} x-\frac{b}{2 \rho_{0}^{2}} \frac{\partial}{\partial x} \\
c^{2}+\frac{(\gamma-1)^{2}\left(\Phi_{1}+Q_{T}\right) T_{0}}{c} \int \mathrm{d} x+\frac{\chi(\gamma-1)^{2} c}{\gamma R \mu \rho_{0}} \frac{\partial}{\partial x} \\
1 \\
-\frac{(\gamma-1) \Phi_{1} T_{0}}{c \rho_{0}} \int \mathrm{d} x
\end{array}\right) \rho_{1}^{\prime} .
$$

In accordance to the roots of dispersion relation (31), (32), the governing equation for sound (20) will include the term $-b /\left(2 c^{2} \rho_{0}\right) \partial^{2} \rho_{1}^{\prime} / \partial x^{2}$ in the left-hand side. The equation for an excess density specific to the thermal mode (23), will be completed by the term $-\frac{\chi(\gamma-1)}{\gamma R \mu \rho_{0}} \partial^{2} \rho_{4}^{\prime} / \partial x^{2}+\frac{(\gamma-1) Q_{T} T_{0}}{c^{2}} \rho_{4}^{\prime}$ in its linear part. It will include also the quadratic nonlinear terms in the right-hand part, proportional to the thermal, viscous attenuation and $Q_{T}$.

\section{Concluding remarks}

The main result of this study is instantaneous equation governing the excess density belonging to the thermal mode, Eq. (23). It is valid in any time for every types of sound, periodic or aperiodic. The only limitations used to simplify calculations, are $c k \tau \gg 1, M \ll 1$, and $|B| \ll k$. The first of them restricts consideration to the high-frequency sound. The nonlinear phenomena by the low frequency sound $(c k \tau \ll 1)$ were studied by the author in the paper [13]. It was discovered there that the attenuation or amplification of sound itself is insignificant. The nonlinear generation of the thermal mode by sound is also insignificant, at least in the field of periodic sound. The study [13] investigates also the nonlinear generation of the third mode, associated with the excess vibrational energy. The conclusions are that it may enlarge efficiently in the field of the low-frequency sound. The acoustic heating (if $B<0$, or cooling, if $B>0$ ) is proportional to $B$, while heating due to standard attenuation is proportional to the overall attenuation due to first, second viscosity and thermal conduction, $b$. The standard thermoviscosity always results to sound attenuation and heating in a nonlinear fluid flow. The larger frequency stipulates the larger thermal and viscous attenuation. The neglecting of standard attenuation comparatively to the nonequilibrium effects are valid at frequencies $\omega \ll\left(\tau \tau_{0}\right)^{-1 / 2}$, where $\tau_{0}$ is the average time of the molecular free pass. On the other hand, $\omega \tau \gg 1$. Both these conditions reduce a domain of sound frequencies where the standard attenuation can be neglected. For $\mathrm{O}_{2}$ at room temperature, $\tau$ equals $10^{8} \tau_{0}$, so that the condition of validity sounds: $10^{4} \gg \omega \tau \gg 1$. Let us note that one can derive the system of conservation equations in the differential form from the gas kinetic Boltzmann equation only under condition $\omega \tau_{0} \ll 1$. Otherwise, the starting point in studies should be the Boltzmann equation. For $\mathrm{O}_{2}$, this condition gives $\omega \tau \ll 10^{8}$, which includes the domain above. Acoustic wave may be attenuated even in nonequilibrium regime if the standard attenuation is enough large; for periodic sound this is conditioned by inequality $\frac{b \omega^{2}}{2 \rho_{0}}+\frac{(\gamma-1)^{2} T_{0}}{2}\left(\Phi_{1}+Q_{T}\right)>0$. 
Detailed studies in dynamics of nonequilibrium gas forces to take into account influence of pumping and heat removal $[3,14]$. With increasing relaxation time the amplification coefficient declines; however, a larger magnitude of pumping $I$ is required to maintain the same degree of nonequilibrium, since $\varepsilon-\varepsilon_{\mathrm{eq}} \approx I \tau$ (Eq. (1)). That makes the nonequilibrium fluids inhomogeneous. The conclusions above are no longer valid in the case of intense pumping, because the linearization should be proceeded with respect to background with non-zero spatial gradients of pressure and density. This alters the very definition of modes. The problem becomes fairly complex mathematically. The paper [14], devoted to the amplification of sound in a flat layer of nonequilibrium gas, reveals some new properties compared to the case of the uniform gas. In particular, the area of instability at the plane pumping intensity - an inverse time of relaxation becomes smaller. Unfortunately, the mathematical difficulties do not allow to consider the problem in general.

\section{References}

[1] Ya.B. Zeldovich, Yu.P. Raizer, Physics of Shock Waves and High Temperature Hydrodynamic Phenomena, Academic Press, New York 1966.
[2] B.F. Gordiets, A.I. Osipov, E.V. Stupochenko, L.A. Shelepin, Sov. Phys. Usp. 15, 759 (1973).

[3] A.I. Osipov, A.V. Uvarov, Sov. Phys. Usp. 35, 903 (1992).

[4] B.T. Chu, in: Nonequilibrium Flows, Ed. P.P. Wegener, Vol. 1, Marcel Dekker, New York 1970, p. 33.

[5] D.F. Parker, Phys. Fluids 15, 256 (1972).

[6] J.F. Clarke, A. McChesney, Dynamics of Relaxing Gases, Butterworth, UK 1976.

[7] O.V. Rudenko, S.I. Soluyan, Theoretical Foundations of Nonlinear Acoustics, Plenum, New York 1977.

[8] S. Makarov, M. Ochmann, Acoustica 82, 579 (1996).

[9] N.E. Molevich, Acoust. Phys. 48, 209 (2002).

[10] N.E. Molevich, Acoust. Phys. 47, 102 (2001).

[11] A. Perelomova, Acta Acustica united with Acustica 89, 86 (2003).

[12] A. Perelomova, Phys. Lett. A 357, 42 (2006).

[13] A. Perelomova, Canad. J. Phys. 88, 293 (2010).

[14] E.V. Koltsova, A.I. Osipov, A.V. Uvarov, Sov. Phys. Acoustics 40, 969 (1994). 